\title{
Integrated Experiment and Modelling of Microstructurally-Sensitive Crack Growth
}

\author{
V.V.C. Wan ${ }^{1, *}$, D.W. MacLachlan ${ }^{2}$, F.P.E. Dunne ${ }^{1}$ \\ ${ }^{1}$ Department of Materials, Imperial College, London, SW7 2AZ, UK \\ ${ }^{2}$ Rolls-Royce plc, PO Box 31, Derby, DE24 8BJ, UK \\ * Corresponding author: E-mail address: v.wan12@imperial.ac.uk
}

\section{Abstract}

An assessment is presented of modelling methodologies which explicitly address microstructurallysensitive crack growth paths in bcc polycrystal ferritic steel. A number of microstructurally differing polycrystal samples are subjected to fatigue and crack nucleation and growth, demonstrating transgranular and intergranular crack paths in characterised microstructures. Microstructurally representative extended finite element crystal modelling, coupled cohesive zone modelling, coupled explicit grain boundary modelling, and plasticity are utilised to assess predicted crack paths against the experimental observations. The incorporation of strong and weak boundary zones when coupled with X-FEM was found to provide quantitative prediction of the transition from transgranular to intergranular cracking and to capture accurately the observed crack paths. Crack tip plasticity was found to have limited effect on microstructurally-sensitive crack path.

Keywords: eXtended Finite Element Method (X-FEM), microstructurally sensitive crack growth, intergranular cracking, transgranular cracking, fatigue 


\section{Introduction}

The progressive development with cyclic loading of deformation structures, damage, crack nucleation and growth leading potentially to material and component fracture remains one of the key life-limiting factors in engineering for safety-critical components. Some components are lifelimited by fatigue crack nucleation processes alone; others by crack propagation in circumstances in which nucleation life is insignificant. For crack development in which both nucleation and growth contribute significantly to overall life, a stage common to all is that when the growing crack may be deemed to be influenced significantly by microstructure. This may be manifested by path tortuosity, in which the direction of growth becomes affected strongly by local microstructural features such as grain boundaries, triple junctions, or second-phases, and by crack growth rate. For the latter, order of magnitude differences may exist for growth along a grain boundary compared to that for cracks propagating through a grain trans-granularly. This paper considers the mechanistic basis and the computational modelling of microstructurally-sensitive crack growth.

Most of today's engineering crack problems are assessed using the fundamental understanding of linear-elastic fracture mechanics (LEFM), where work from Griffith [1] and later by Irwin [2] led to the stress intensity factor associated with cracks. Fracture mechanics provided a platform for many developments of software tools and finite element packages for crack propagation simulations such as FRANC2D/3D [3, 4], BEASY [5], ADAPCRACK2D/3D [6, 7] and ZENCRACK [8]. However most of these packages require extensive continuous re-meshing to capture geometric discontinuity during subsequent crack advancements which can be computationally expensive. There are some techniques which do not require the need for remeshing including the extended finite element method (X-FEM) which has been demonstrated by Sukumar et al. [9-13] in their 2D and 3D models. Similar to X-FEM are other possibilities such as that presented by Chen et al. [14] which introduced a concept called the floating node method. More recent methods include mesh-free approaches [15, 16] where Barbieri et al. [15] introduced new weight-functions for node enrichment and Nguyen et al. [17] demonstrated the potential of phase field methods to simulate crack nucleation and propagation.

Though LEFM provides solutions for long crack growth problems, it is known from early reviews [18, 19] that the basic LEFM approach fails to correlate crack growth rates at the microstructural length scale due to significant influences of local morphology, crystallography and the microstructural features affecting both elastic and plastic behaviour local to the crack front. Reviews (e.g. [20]) have demonstrated the importance of mechanistic approaches to address microstructural-level deformation and crack nucleation including the consequences of crystal orientations, grain 
morphology, and the establishment of dislocation structures. Micromechanical modelling of short fatigue cracks is also of current interest and has recently been reviewed in [21] and authors have introduced empirical, analytical or complex numerical solutions to address local phenomena and crack tip behaviour.

At the microstructural level a number of modelling techniques have been used to introduce discrete local separation behaviour. The boundary element method was used in Christ et al. [22] along slip bands of interest where they looked at conditions for propagating cracks in duplex steel. A popular approach is that of cohesive zone modelling [23-27], where traction-displacement relationships are typically applied between grains as cohesive interfaces. Kupka et al. [27] also adopted a cohesive zone approach to describe grain boundary separation based on a normal traction stress criterion coupled with fracture energy for crack initiation in their micro-cantilever crystal plasticity model. An alternative technique involves a non-local damage method [28-30] which calculates the degradation of the material stiffness to model damage and fracture where some author [29] have combined the method with local crystal plasticity to investigate microstructural-sensitive crack nucleation and propagation. One advantage with these methods is the flexibility to integrate user specified criteria illustrated by Vajragupta et al. [31] for cleavage fracture in their dual-phase steel, where the martensitic 'phases' employed a maximum principal stress criterion, and ductile damage of the ferrite phase employed a criterion based on stress triaxiality and equivalent plastic strain. However, some have recognised the potential of X-FEM exclusively to model microstructure-sensitive crack growth. Sukumar et al. [32] introduced brittle fracture parameters and toughness ratio effects for crack propagation in their $2 \mathrm{D}$ microstructure, taking into account both intergranular and transgranular microstructural cracks. The criteria were also considered by Farhangdoost et al. [33] where the crack growth parameters were fitted to match with their experimental Ti-6Al-4V microstructural crack observed. Menk et al. [34] also utilised X-FEM to model cracks emerging along boundaries in solder joints where equivalent creep strain was used as a criterion for boundary cracking. The ranges of methods and growth criteria used reflect the continuing interest to better address local microstructurally-sensitive crack growth phenomena.

The research literature demonstrates X-FEM has been shown to have great facility to capture crack growth without prior knowledge of crack path and without the need for re-meshing. Importantly, XFEM also benefits by providing a reduction in computational expense and complexity due to its availability in commercial Abaqus/Standard FE software. This paper presents a systematic study which utilises X-FEM to address the influences of microstructure particularly on crack path and tortuosity and includes assessment of the role of local material behaviour (including isotropic 
elasticity, anisotropic elasticity and isotropic plasticity). In addition, cohesive layers and grain boundary zones are also introduced in combination with X-FEM to enhance the capability of capturing experimentally observed crack paths. A significant feature of this work is that a range of microstructurally differing two-dimensional, prismatic grain structured ferritic steel polycrystals subjected to four-point bending are available, providing direct and unambiguous comparisons between model capabilities and experimental observations. Hence details of each cracked sample and the model representations have explicit microstructural grain reproductions from full experimental EBSD characterisation. These experimental data have also been used in previous studies $[35,36]$ which address fatigue crack nucleation.

\section{Modelling methodologies}

The methodologies described briefly in this section in the context of microstructure-sensitive crack growth include X-FEM, X-FEM combined with cohesive layers, and X-FEM with grain boundary zones employed within Abaqus/Standard.

\subsection{Extended finite element method (X-FEM)}

The X-FEM method eliminates the need for re-meshing during the course of moving discontinuities in a model as the mesh is not bounded by conventional finite element boundaries. X-FEM is simply an FE method where typical displacement based approximations are enriched with features by utilising the concept of partition of unity [37]. The X-FEM formulation within Abaqus finite element analysis (FEA) is summarised in $[9,38]$. Additional developments to the X-FEM library include consideration of higher-order displacement functions in the enrichment feature to compensate for complex crack problems [39].

X-FEM nominally allows automated solution-dependent crack growth in which the crack propagation path may be modelled independently of the model mesh by incorporating an enriched displacement vector $\boldsymbol{u}$ is expressed as

$\boldsymbol{u}^{h}(x)=\sum_{i} N_{i}(x) \boldsymbol{U}_{i}+\sum_{j \in J} N_{j}(x) H(x) \boldsymbol{a}_{j}$

where $N$ are the shape functions, $\boldsymbol{U}$ the displacement vector, $H(x)$ the Heaviside jump discontinuous function equal to +1 on one side of the crack and -1 on the other, $\boldsymbol{a}_{j}$ is the additional nodal enriched degree of freedom vector. The Heaviside function is applied to the set of nodes $j$ whose shape function support is cut by the crack interior, and the other remaining node sets $i$ in the model follow the conventional classical finite element method formulation. Further detail of the numerical solution can be found in $[13,32]$. 
The X-FEM Abaqus/Standard model follows a cohesive segment approach and provides crack failure criteria for growth which include those based on principal or nominal stress or strain, traction or separation. Shi et al. [40] note from their short crack propagation study on fatigue tested DS Ni-base super alloy, that microstructural crack propagation initially occurs at sites where maximum principal stress was highest, and the crack growth path turned to the plane perpendicular to the loading direction. In the present X-FEM studies, the maximum principal stress criterion is also employed to model crack propagation in our 2D prismatic grain structured ferritic steel polycrystals. Following Abaqus/Standard nomenclature, the crack failure criterion can be represented [37] as

$f=\frac{\left\langle\sigma_{\max }\right\rangle}{\sigma_{\max }^{0}}$

$1.0 \leq f \leq 1.0+f_{\text {tol }}$

where $\sigma_{\max }^{0}$ is the critical maximum allowable principal stress, \langle\rangle is the Macaulay bracket with the usual interpretation, and $f$ is the fracture criterion for which the crack is extended when Eq. (3) is satisfied, and where $f_{\text {tol }}$ is the fracture tolerance, here set to be 0.05 . The critical maximum principal stress is introduced arbitrarily and is used consistently throughout the X-FEM crack propagation failure analysis presented. A second energy-based criterion was also introduced to define the evolution of damage until eventual failure. The XFEM damage energy refers to critical energy release rate for fracture, which can be calculated from the fracture toughness. Once the failure criterion is satisfied, the crack is extended perpendicular to the maximum principal stress direction which is integrated within Abaqus/Standard. This technique is the focus of the method to model microstructure-sensitive crack growth in the present work, which is facilitated to address intergranular and intragranular cracking in our 2D polycrystals, and the results are compared directly with experimental observations in ferritic steel samples using scanning electron microscopy (SEM).

\subsection{Cohesive Zone Modelling}

Cohesive zone modelling is considered in some fracture studies where separation of surfaces is incorporated through discrete traction behaviour, facilitating flexibility for local specified behaviour across pre-defined interfaces. Abaqus/Standard offers a library of interaction modules which allow the user to specify generalised traction-separation behaviour on selected cohesive surfaces. However, it is possible using cohesive elements to include material behaviour of finite thickness using the continuum approach. For both cohesive surfaces and cohesive elements, a linear elastic (or more complex) traction-separation law may be employed. The traction for the case of a cohesive surface interaction is given by 
$\mathbf{t}=\left\{\begin{array}{l}\mathrm{t}_{n} \\ \mathrm{t}_{s} \\ \mathrm{t}_{t}\end{array}\right\}=\left[\begin{array}{lll}K_{n n} & K_{n s} & K_{n t} \\ K_{n s} & K_{s s} & K_{s t} \\ K_{n t} & K_{s t} & K_{t t}\end{array}\right]\left\{\begin{array}{l}\delta_{n} \\ \delta_{s} \\ \delta_{t}\end{array}\right\}=\boldsymbol{K} \boldsymbol{\delta}$

where $\mathbf{t}$ is the nominal traction stress vector with subscript $n$ as the normal component, and $s$ and $t$ are the two shear traction components. The nominal stress vector is dependent on the separation $(\boldsymbol{\delta})$ with respect to the normal, tangential and shear stiffness components in 3D analysis, which need to be defined for uncoupled traction-separation behaviour. Non-diagonal terms are included for a coupled analysis. The interface stiffness values may be selected in order to attempt to replicate experimental observations, although their mechanistic bases potentially remain elusive. Nonetheless, the cohesive surface methodology may be employed concurrently with X-FEM.

\subsection{Boundary zones}

Grain boundary based techniques are considered in which finite thickness boundary zones are defined and modelled using continuum solid elements for which grain boundary property specification then becomes possible, either through use of Abaqus standard material models or user defined material models. In the present study, boundary zones are employed at selected microstructural features to assess local crack behaviour.

An example of this is shown in Fig. 1 where a boundary zone (in white) is shown schematically with respect to grain B. This methodology when coupled with X-FEM then enables investigation of microstructure-sensitive crack propagation as grain boundary properties are varied in order to give, for example, elastically stiff through to compliant boundary properties. The computational crack growth studies may, again, be compared quantitatively with experimental SEM observations.

\section{Material, fatigue samples and modelling}

Experimental fatigue tests have been conducted on notched specimens as failure sites are focused around the region of the notch with high stress concentrations. In polycrystal specimens, small crack growth may be very dependent on the microstructure and this is evident at nano-scale [41] to micron-level [40,42] and through to larger millimetre sized grains [35, 36]. For micron-level studies, it is difficult to monitor fatigue crack growth with good resolution and some authors $[40,42]$ have addressed microstructural fatigue scatter by considering crack growth direction angle with respect to the notch bisector. However, with larger-grained specimens, clearer detailed crack growth paths can be better captured with SEM as shown in ferritic steel notched polycrystal specimens [35, 36]. Hence in this paper, large-grained, two-dimensional prismatic grained notched samples are considered as originally reported in $[35,36]$. 
The results of fatigue tests on body centred cubic $(B C C)$ prismatic single phase ferritic steel samples are reported in $[35,36]$. The composition of the ferritic steel is listed in Table 1 . The grains are relatively large with an average size of around $1850 \mu \mathrm{m}$ and back-scattered electron imaging of the $\alpha$-Fe single phase alloy shows it contains a distribution of TiN precipitates (Fig. 2) with size of about $5 \mu \mathrm{m}$. The TiN precipitates accumulate a total $0.1463 \%$ composition in mass within the SUS430LX alloy (see Table 1).

The ferritic steel specimens were loaded under four-point bending configuration experimentally and a model geometry finite element representation has been generated for each (differing) microstructure of interest. Specimen geometries are shown in Fig. 3 together with an example microstructure and the finite element microstructural representation. Within the notch region, the full explicit grain morphology and crystallographic representation is modelled and outside this key region of interest, isotropic elastic material behaviour is assumed for simplicity. First-order solid continuum elements are utilised for the purposes of X-FEM studies where on average each grain is filled with 1,390 elements. A closer look at the finite element mesh is shown in Fig 3. All 2D models are generated to follow as close a representation as possible of the prismatic grain structure defined for the ferritic steel polycrystal specimens and hence mode I crack behaviour is the focus of our crack growth studies.

Six selected ferritic steel notched specimens are considered in this paper to study the influences of differing grain morphology and crystal orientation on microstructure-sensitive crack growth. The clear differences between the six microstructural morphologies are evident from Table 2 and each sample is identified and labelled. The experimental SEM crack growth path observations are presented in Table 3, and the experimental fatigue test conditions and cycles to failure for each notched specimen are labelled correspondingly where load applied ranged from $400-460 \mathrm{~N}$ at $15 \mathrm{~Hz}$ frequency. Full details of the individual grain crystal orientations have been obtained using electron backscatter diffraction (EBSD) which are represented through Bungee convention and are listed in Appendix A. Monotonic direct load control is imposed onto the beam at the location of the two vertical arrows as shown in Fig. 3 to induce progressive crack development and growth within the notch region. The primary focus in this study is crack path and its microstructural sensitivity. For this reason, and because the modelling of cyclic loading continues to be computationally expensive (particularly with combined X-FEM and plasticity material behaviour), the loading in the modelling is confined to being monotonically increasing. However, failure by crack advancement under fatigue or monotonic loading in early studies $[43,44]$ has been shown to be similar, but naturally crack growth rate and the stress intensity differ. Previous notched specimen studies $[35,36]$ have 
demonstrated the important roles of elastic anisotropy and crystallographic slip in fatigue crack nucleation. However, it is not yet understood clearly how strain at the microstructural level influences fatigue cracking paths and how cracks may exhibit sensitivity to slip field variation. Carroll et al. [45] provided detailed insights of full-field measurements of plastic strain near growing fatigue cracks, for which asymmetric strain lobes formed closely associated with past crack tip plastic zones. Plastic strain field development around crack tips has consequently led some authors $[46,47]$ to assess and utilise accumulated plastic strain as the critical parameter for crack growth development in their studies. The study in [48] incorporated crystallographic slip in their modelling, and crack tip stresses were found to remain largely independent of crystal orientation and the local slip established, but plastic zone size and shape did depend upon it. Hence, in the crack modelling studies reported here, the maximum principal stress and its direction are considered as the criteria adopted and assessed to determine the experimentally observed crack growth directions within the X-FEM crack propagation model.

Based on the six ferritic steel samples with differing microstructural morphology listed (Table 2), use of X-FEM together with representation of the microstructure then enables predicted crack paths for the loaded samples to be established and compared with the experimental observations. The models allow a systematic study of the roles of elastic anisotropy, plasticity and grain boundary effects to be evaluated in the context of predicted crack path in the light of the experimental observations. The results of these studies are presented in the next section, in which X-FEM with grains represented as elastically isotropic and anisotropic crystals are assessed first. This is followed by an assessment of cohesive surfaces to represent grain boundaries followed by explicit grain boundary modelling utilising solid continuum elements.

\section{X-FEM results and discussion}

In this section, systematic studies using X-FEM and X-FEM coupled with cohesive or boundary zone techniques to model microstructure-sensitive crack growth in six ferritic steel specimens are presented. The influences of morphology and material properties are considered and results are compared directly with known experimental crack paths taken from SEM images. Firstly, for a particular sample, only isotropic elastic material behaviour is considered (to provide a simple benchmark) and thereafter the role of anisotropic elasticity is compared with resulting microstructural cracks presented for all six ferritic steel samples. Isotropic plasticity is then included and assessed for a further particular sample. For all model samples, an initial average crack nucleation length of $40 \mu \mathrm{m}$ is introduced at the location of experimentally known crack nucleation sites with a critical energy release rate for fracture of $50 \mathrm{~N} / \mathrm{mm}$. Local microstructural crack 
behaviour is discussed, particularly transgranular and intergranular microstructural crack growth. Cohesive and boundary zones are integrated with X-FEM in order to capture some of the more complex intergranular crack growth paths witnessed which were not well captured by the simpler models.

\subsection{Isotropic elasticity}

Based on the model generated with reference to Fig. 3 (Sample A shown in Table1), full isotropic elasticity is assigned to all eleven grains in the sample where each grain is assigned the nomenclature A-K as described in Table 2. Note, therefore, that for the case of elastic isotropy, the polycrystal sample simplifies in fact to a homogeneous elastically isotropic notched beam in which crack growth normal to the local maximum principal stress is expected initially, and which ultimately becomes normal to the remote applied (bending) stress once the influence of the notch stress state has diminished sufficiently. An initial crack is introduced as shown in Fig. 4(a) at the grain triple junction near the left of the notched section where crack nucleation is identified from the SEM image (shown in Fig. 4(e)). Once the crack nucleates, the experimental fatigue test shows the crack grows at an angle of about $-4.5^{\circ}$ away from the $\mathrm{Y}$ axis and there is little change to the direction as it grows deeper into primary grain D. (Note that a $0.0^{\circ}$ crack growth direction refers to growth straight vertically downwards.) Once the crack approaches grain boundary D-K (grain boundary between grains $\mathrm{D}$ and $\mathrm{K}$ ), there is a small change of angle but the crack grows straight through the boundary into grain $\mathrm{K}$ at an angle of $-5.5^{\circ}$ from the $\mathrm{Y}$ axis. At the bottom of the SEM image shown in Fig. 4(e), as the crack continues to develop further in grain $\mathrm{K}$ a kink develops and deviates to about $-70^{\circ}$ away from the $\mathrm{Y}$ axis and towards grain boundary $\mathrm{K}-\mathrm{J}$.

The X-FEM predicted crack path using isotropic elasticity during the first increment of loading with the initial crack in place is shown in Fig. 4(b) and shows the axial X-stress distribution in the notched region. As the crack develops and grows midway into grain $\mathrm{K}$, shown in Fig. $4(\mathrm{c})$, the crack tip presents a symmetrical stress field where highest stress is naturally concentrated around the region of the crack tip. The predicted crack deviates at an angle of $-8.0^{\circ}$ from the $Y$ axis or perpendicular to the nominal maximum stress $\sigma_{m}$ direction, because of the stress state developed by the notch. This is clearly different to the experimental crack propagation direction of $-4.5^{\circ}$. The complete predicted crack profile is shown in Fig. 4(d) and it becomes apparent that once the crack grows deeper into grain $\mathrm{D}$, removed from the influence of the notch, the nominal stress direction $\sigma_{m}$ governs the crack path which becomes parallel to the $Y$ axis direction. No influence of the grain boundary D-K is anticipated since in this analysis, all 'grains' are elastically isotropic. 
The elastically isotropic prediction naturally therefore fails to capture the experimentally observed crack growth path within the polycrystal and it is apparent that the local, grain-level anisotropy is potentially important in driving the crack path. Hence this is addressed next.

\subsection{Anisotropic elasticity}

With reference to sample $A$, full incorporation of crystallographic information listed in Appendix $A$ is assigned to the sample A model and X-FEM is used to calculate crack propagation behaviour, as before. Here grain boundaries are defined simply as the demarcation between two grains with differing crystallographic orientations.

Fig. 5(a) displays the axial stress distribution early in the loading before significant crack growth and it is apparent that the grain-level elastic anisotropy greatly modifies the stress distribution. Greater heterogeneity of stress is developed across the notched region and as the X-FEM crack advances into primary grain D shown in Fig. 5(b), (mildly) non-symmetric stress fields ahead of the crack tip are observed. At this stage, the model crack propagates at about $-4.0^{\circ}$ from the $Y$-axis direction, which is very close to the observed experimental crack direction. The final X-FEM microstructural crack path as shown in Fig. 5(c) shows the crack grows with little deviation from the initial crack direction and hence with limited influence from the stress state developed by the notch and in fact grows straight through grain $\mathrm{D}$ into grain $\mathrm{K}$ with minimal deviation. This is the crack growth direction observed experimentally (Fig. 4(e)) and it is interesting to note the very similar crack kinking seen in the same proximity within secondary grain $\mathrm{K}$, highlighted in yellow in Fig. 5(c). The elastic anisotropy for sample A shows some influence on the crack growth path when compared with the isotropic elasticity crack path labelled ' 1 ' in Fig. 5 (d) where the differences are clear and the elastically anisotropic X-FEM crack labelled ' 2 ' compares well with the experimental observation. To understand further the differences between the two crack paths taken, the X-FEM crack propagation criteria used are based on the maximum principal stress (Eq. (2) and (3)) and rules that the crack direction grows perpendicular to the local maximum principal stress direction.

Fig. 6(a) shows early crack growth, for elastically isotropic 'grains', into primary grain D and the maximum principal stress directions (double-ended arrows) around the vicinity of the crack region. The size of the principal stress direction arrows is indicative of the magnitude of the maximum principal stress (longer the arrow, higher the maximum principal stress locally), and the principal stress direction corresponding to largest magnitude of maximum principal stress (marked with dashed line) is located at the crack tip region and is inclined at $8.0^{\circ}$ from the $\mathrm{X}$-axis, hence giving rise to the crack growth direction of $-8.0^{\circ}$ seen in the model studied in Fig. 4 for isotropic elasticity 
conditions. Similarly, under anisotropic elasticity conditions, the largest magnitude of maximum principal stress direction marked on Fig. $6(\mathrm{~b})$ shows a lower inclination angle of $4.0^{\circ}$ from the horizontal X-axis compared to the isotropic elasticity solution in Fig. 6(a). This results in the final crack growth path in Fig. 5(c) which is near-identical to the experimental crack path in Fig. 4(e).

Elastically anisotropic polycrystal modelling has next been carried out for all of the experimental polycrystal samples shown in Table 2, in order to obtain all of the predicted crack paths and they are shown collectively in Table 3 together with the corresponding experimentally observed paths obtained through SEM imaging.

From Table 3, the crack path modelled in sample D also shows close agreement to the SEM crack path captured. Across the primary grain A (refer to Table 2 for sample D), crack directions in both the model and experimental observation deviate towards the left of the notch and the crack grows through grain boundary A-G and then further grows across grain G. Kinking was also interestingly captured in the model midway through grain $G$ which is also present experimentally. The microstructural crack path developed for Sample C is slightly more complex as SEM imaging shows crack nucleation just to the right of the notch and growth towards the left in primary grain $\mathrm{B}$. The direction of the crack path is closely captured in the model which also propagates towards the left of the notch, but greater deviation is witnessed experimentally with a difference of $13.0^{\circ}$ between the model and known crack direction. As the crack grows into secondary grain $A$, the crack direction changes to closer to the vertical which is similarly observed in SEM. Subsequent model crack growth into the third grain (grain $\mathrm{G}$ ) predicts crack deviation towards the left of the grain which is contrary to experimental observation which shows growth to the right. However, with respect to Table 3, it should be noted that the regions shown for which microstructural modelling has been carried out are not the complete loaded beam and that adjacent to the microstructural regions, isotropic elasticity has been utilised in the model beams. Hence, as model cracks approach and grow close to the bottom boundary, their paths are likely to differ from the experimental observations so that the predicted response becomes unreliable in this vicinity.

The SEM images of crack growth for samples B and $\mathrm{F}$ both show strong influence of microstructural features (notably grain boundaries) on crack path, giving rise to both intergranular and transgranular crack growth characteristics in the samples. For sample B, the SEM-observed crack growth direction after nucleation in primary grain I (see Table 2) grows at an angle of $-19.0^{\circ}$ from the nucleation point, which was also captured well by the elastically anisotropic X-FEM crack propagation model. Once the experimentally-observed crack cuts though the primary grain and hits the grain boundary I-M, very significant crack path deviation of $80.0^{\circ}$ is observed where growth continues along grain boundary I- 
$\mathrm{M}$ and into neighbouring grain boundary $\mathrm{M}-\mathrm{N}$. This intergranular crack behaviour was not captured with the X-FEM model by which the crack is predicted to grow straight across grain boundary I-M and into neighbouring grain $\mathrm{M}$. This behaviour is somewhat similar to that observed for sample $\mathrm{F}$. The initial crack in the experiment nucleates to the left to mid-section of the notch near grain boundary B-F and grows to the left of the primary grain F reaching grain boundary E-F with further growth along the grain boundary. As the crack develops and reaches the triple junction in the microstructure, the SEM image shown in Table 3 shows intergranular crack growth extending into boundary K-F, which at the end of the grain boundary deviates $60.0^{\circ}$ back into grain K. The X-FEM crack propagation model shows similar microstructural short crack behaviour within the primary grain $\mathrm{F}$ and close to grain boundary E-F. But as the crack reaches close proximity to the triple grain boundary junction, there was found to be no driver for the crack to grow along grain boundary K-F, and instead develops straight into grain $\mathrm{K}$.

In the other samples, only Sample E (in addition to B and F) showed significant differences of crack growth paths between model predicted and experimental results, as seen in Table 3. The SEM image shows the crack grows an average $+34.0^{\circ}$ from the $Y$-direction whereas the model predicts a path of $+11.0^{\circ}$. The experimental SEM image further reveals the crack cuts through grain boundary D-K, and consequently grows into the direction of the triple junction between grains $\mathrm{K}$, $\mathrm{J}$ and $\mathrm{M}$ just before which, the crack grows briefly in the direction along neighbouring grain boundary K-J. However, in the model prediction, the crack avoids penetrating grain boundary $\mathrm{D}-\mathrm{K}$ and instead deviates to cut through grain boundary D-J and grows into grain J. The differences suggest a role played by grain boundaries in crack growth path development; in the experiment, grain boundary D-K seems favourable for the crack to penetrate, but the model does not physically address this and instead the principal stress driver leads to the avoidance of the grain boundary completely. Although the general growth direction is captured (path to the right of the notch), the large differences in crack growth path could result from other microstructural features not properly represented in the model. For example, plasticity effects and local BCC slip activity, strongly dependent on crystallographic orientation, have not been included.

The initial axial stress distribution shown in Fig. 7(a) for sample F shows highly localised regions of stress at the notch surface as expected and noticeably higher stress to the left of the notch due to the initial crack present. As the crack grows towards the triple junction, a non-symmetric crack tip stress distribution is shown in Fig. 7(b) with inset local to the crack tip. Here higher stresses are developed to the right along grain boundary K-F where the experimentally-observed crack in fact grows, rather than across the neighbouring grain boundary K-E. The Mises stress distribution shown 
in Fig. 7(c) also suggests a similar story, and greater localised stresses are developed in grain $\mathrm{F}$ behind the grain boundary K-F and smaller localised peak stresses are present in the neighbouring grains.

In summary, based on the anisotropic elasticity X-FEM crack propagation approach, of the six ferritic notched polycrystals modelled and summarised in Table 3, three samples (,$C$ and $D$ ) showed good agreement of microstructurally-sensitive crack path between model and experiment if not identical (for samples $A$ and $D$ ); one sample (E) showed poor agreement and for two further samples (B and $F$ ), crack growth path in the primary grain was well predicted compared to experiment but the X-FEM model could not capture the intergranular crack growth witnessed in experiment. As these two model samples showed that the X-FEM model with elastic anisotropy did not capture intergranular crack growth features, nor the stress states necessary to drive intergranular crack propagation (as detailed in Fig. 7), it is therefore of interest to examine other aspects of the microstructure in more detail and in this study, grain boundaries are examined further. In order to do this, examination is now focused on sample $\mathrm{F}$ which, from Table 3, can be seen to show much intergranular growth. Firstly, the role of plasticity is investigated, and followed by consideration of grain boundary properties and behaviours.

\subsection{Elastically anisotropic and plastically isotropic grain modelling}

The X-FEM anisotropic elastic crack propagation results (Table 3) for sample F did not feature intergranular crack development as observed in the experimental test results. Isotropic plasticity with linear strain hardening is introduced to assess whether any improvement of predicted crack paths may be obtained. Crack growth to an extent has some form of dependence on plastic deformation in regions near the crack tip where stresses exceed the yield stress, potentially influencing stress state development and crack path. Stress relaxation due to the local deformation within the plastic zone redistributes the stress fields to satisfy equilibrium conditions. Hence incorporation of plasticity is assessed to investigate whether the redistribution of stresses affects the local crack growth in polycrystal sample F using X-FEM. The plasticity properties are obtained from previous stress strain data $[35,36]$ where the shear stress in yield is set to $85 \mathrm{MPa}$ with hardening modulus of $2.2 \mathrm{GPa}$.

Details of the axial stress distribution for crack propagation up to grain boundary K-F are shown Fig. 8 (a) obtained from the enhanced XFEM model. Inhomogeneity resulting from elastic anisotropy is again captured throughout the polycrystal model, in a similar way to that shown in Fig. 7(b). With the same stress range illustrated, the von Mises crack tip stress field shown in Fig. 8(b) shows a 
somewhat different high stress zone in comparison to Fig. 7(c) when plasticity is not considered. At the crack tip (Fig. 8(b) zoomed window), a broader butterfly spread of highly localised stresses is developed on both sides of the neighbouring grain boundaries K-F and E-F locally, whereas Fig. 7(c) previously studied shows a region of high stress simply behind grain boundary K-F. The predicted equivalent plastic strain distribution is shown in Fig. 8(c) where regions of plastic straining are only observed local to the crack tip. Once the X-FEM crack propagates close to the triple junction and hits grain boundary K-F, significant build-up of plasticity is present with maximum of 0.371 recorded just behind the grain boundary. Nevertheless, the X-FEM crack propagates straight through the grain boundary.

The final predicted crack growth path is shown in Fig. 8(d) and when compared with the anisotropic elasticity X-FEM solution in Table 3 (i.e. no plasticity) - sample F, shows no improvement in capturing the experimentally observed intergranular local crack growth. Though plasticity plays a role and affects the crack tip stress distributions and likely crack growth rate, the effect on crack path is here found to be small. Farukh et al. [46] have considered a local dislocation plasticity based constitutive rule in their crack growth studies which have provided further insight into plasticity affects on crack growth rate, but it is still an aspiration to understand how dislocation based plasticity may influence microstructural crack path development. Some authors have argued intergranular stresses play a significant role in the failure process [49], but the development of residual grain boundary stresses was not found to be highly correlated with the plastic strain in close proximity to the boundary and the soft-hard grain combinations. The role of grain boundaries in influencing and inhibiting crack growth remains of considerable interest. For this reason, focus is next given to grain boundary properties and behaviours, firstly through consideration of cohesive separation interfaces considered within the X-FEM crack propagation formulation.

\subsection{Anisotropic elasticity with cohesive interfaces in X-FEM}

It was found above that accurate representation of morphology and crystallographic orientation (through specification of elastic anisotropic stiffness) integrated within X-FEM was not able to capture the intergranular cracking witnessed in SEM images of Sample F (Table 3). We therefore examine the possibility of capturing intergranular cracking by use of cohesive interfaces and interactions incorporated along specific grain boundaries of interest. Because the incorporation of isotropic plasticity was found to have minimal effect on the X-FEM crack propagation path solution when compared with anisotropic elasticity only, for simplicity and consistency, isotropic plasticity is now neglected. Earlier X-FEM crack propagation solutions demonstrated that crack development in 
the primary grain $\mathrm{F}$ was captured well together with some grain boundary crack growth captured along grain boundary E-F, but cracking along grain boundary K-F was not captured.

To introduce cohesive interfaces, cohesive traction-separation behaviour is specified along the entire grain boundary around primary grain F, and a single X-FEM crack domain is defined in the primary grain. The traction-separation behaviour employed is given by Eq. (4), but for simplicity, the off-diagonal terms are taken to be zero (i.e. no mode coupling). The in-plane normal and tangential stiffness components $K_{n n}$ and $K_{S S}$ are set to 5.0GPa and 4.0GPa respectively for uncoupled tractionseparation behaviour. Introducing X-FEM crack propagation to initiate at the site of crack nucleation, the predicted crack grows up to grain boundary K-F, and the notched region axial stress distribution is shown in Fig. 9(a) which has similar overall form to that obtained without cohesive zone modelling shown in Fig. 8(a). Locally, at the crack tip, high stresses are concentrated within the primary grain F as shown on Fig. 9(a) - top left inset. Separation is witnessed along the grain boundary K-F once the $\mathrm{X}$-FEM crack reaches the cohesive interface, introducing high stress concentrations and provoking cohesive separation as shown in Fig. 9(b). A closer look at the deformed state separation is shown in the top right inset in Fig. 9(b). During the first indication of separation along grain boundary K-F, the axial stress distribution (inset in Fig. 9(a)) shows the displacement of the crack tip stress field along grain boundary K-F and into grain K. Introducing cohesive interfaces, separation along the whole length of the grain boundary K-F is subsequently captured (Fig. 9(b) zoomed window) and hence in this way, the entire transgranular and subsequent intergranular crack growth observed in the experiment for sample $\mathrm{F}$ is reproduced by the coupled $\mathrm{X}$-FEM-cohesive zone model as shown in Fig.9(c).

The experimental observations show that the intergranular crack then diverts again into grain $\mathrm{K}$ becoming transgranular. However, the cohesive interaction is achieved by appending two domains in the model and specifying the respective surface-surface contacts, but the two domains remain separate in the finite element context, and this prohibits the subsequent use of X-FEM which is incompatible with multi-domain continuous crack propagation after cohesive separation. A potential methodology to overcome this problem is the introduction of a crack initiation criterion to provoke cracking in the second domain, but this may well require extensive material modelling behaviour to capture and address local microstructurally sensitive parameters [36]. Hence the use of cohesive interfaces currently renders transgranular crack modelling difficult after intergranular cracking is captured in the model. A further alternative is to address the behaviour of the grain boundaries using solid continuum elements (as opposed to cohesive zones) as this potentially facilitates continuous XFEM crack propagation through or along microstructural features (intergranular and/or 
transgranular cracks) without limitation and enables control of the grain boundary and interface properties. This is addressed next.

\subsection{Anisotropic elasticity with boundary zones}

As cohesive interfaces introduce complications for further crack propagation beyond the primary grain, boundary zones are proposed to benefit the flexibility of a continuous X-FEM crack propagation in the microstructure. By introducing a zone of independent properties along the grain boundary interfaces of interest, it is hypothesised that the grain boundary properties may be defined to provoke the growth of intergranular cracking, known to occur experimentally in samples $\mathrm{B}$ and $\mathrm{F}$ in Table 3. In the present work, the boundaries are taken to be elastically isotropic and consist of a single element thickness of $20 \mu \mathrm{m}$. 'Strong' and 'weak' (more strictly, stiff or compliant) grain boundary properties are investigated and results compared. Here, weak and strong grain boundaries have been assigned to have 100x smaller and greater modulus respectively than the equivalent ferritic grain isotropic modulus.

For sample $F$, grain boundary zone elements were exclusively introduced along grain boundaries K-F and E-F where intergranular cracking is known to occur along these interfaces. The results are shown in Fig. 10. Figs. 10(a) and Fig. 10(d) are the resulting axial stress distributions with weak and strong grain boundaries respectively when the X-FEM crack has developed up to grain boundary K-F. Localised stresses develop along the grain boundary and close examination of the crack and grain boundary region shows the grain boundary element interface has considerably lower stresses for the weak grain boundary (Fig. 10(a) - black arrow indicating grain boundary location) in comparison with the neighbouring grains. In contrast, when the strong grain boundary zone is introduced, very high stresses are developed along the grain boundary. When considering the final X-FEM crack growth path obtained, it is seen that the weak grain boundary (Fig. 10(b) and Fig. 10(c)) leads to minimal cracking along the grain boundary and the crack simply cuts through the grain boundary into grain $\mathrm{K}$. However for the strong grain boundary (shown in Fig. 10(e) and Fig. 10(f)), the crack is seen to follow the grain boundary path K-F for longer. However, nonetheless, the crack again is seen to deviate away from the grain boundary and in to grain $\mathrm{K}$ at about one-third of the length of grain boundary $\mathrm{K}$ F. This remains early compared to the experimental SEM crack observation for Sample F.

Sample B has also been analysed with inclusion of grain boundary zone modelling in an attempt to capture the experimentally observed intergranular cracking in this sample. For this sample also, the transgranular cracking could not be captured using anisotropic elasticity with X-FEM alone. Grain boundary zones were introduced along grain boundaries I-M and M-N where intergranular cracking 
is observed in the experiment. In this instance, the boundary zone modulus has been magnified and set to $1000 x$ higher or lower than that for the ferritic grain. The X-FEM and grain boundary zone results for predicted crack path are shown in Fig. 11. As the crack advances towards the grain boundary I-M as shown in Fig. 11(a) and Fig. 11(d), localised high axial stresses develop at the grain boundary, resulting from the interaction of the crack tip stress field with the grain boundary. For the grain boundary zone with weak stiffness in sample B, Fig. 11(b) and Fig. 11(c) shows that the boundary interaction did not provoke intergranular cracking and instead the crack is seen to grow into grain $\mathrm{M}$ with significant crack path deviation towards the left of the grain. For strong grain boundary stiffness properties as shown in Fig. 11(e) and Fig. 11(f), crack growth along the grain boundaries I-M and M-N was well captured for which the SEM images for Sample B in Table 3 show very similar microstructurally-sensitive crack growth behaviour. The model predictions indicate some kinking in the crack path in grain I which was not observed in the experiment (Table 3) for Sample B. However, it has been shown for the more complex, contorted crack growth paths in Samples B and F for which both transgranular and intergranular growth is observed, the combined grain boundary property modelling with X-FEM has provided significantly improved predictions of experimentally observed complex crack paths over those from the simpler methodology of incorporating crystallographic orientation with X-FEM through anisotropic stiffness, and has considerable advantages over coupled X-FEM and cohesive zone modelling.

Strong grain boundary stiffness properties describe microstructural transgranular cracking behaviour better as a result of the higher stresses generated along the boundaries, thus provoking preferential crack growth along the grain boundary itself rather than deviating to become transgranular. The development of high concentration of stresses along grain boundaries has been observed experimentally by Jiang et al. [50] in an EBSD study which shows highest values of the maximum inplane shear stress tend to be found close to grain boundaries. These measurements were made, however, in the absence of the presence of cracking. Clearly, the assumption that grain boundaries may be modelled as elastically isotropic is simplistic, and their behaviour is likely to be much more complex. In addition, the activation of slip at the crack tip, and its interaction with grain boundaries, potential transfer or build up and contribution to local stresses, are all potentially important. But results presented earlier indicate that at least in the context of crack growth driven by maximum principal stress, the presence of plasticity leading to local stress relaxation and redistribution does not overly influence the crack growth path. Some studies [51] have recognised grain boundaries as key features for fatigue crack growth resistance and have considered slip crystallography as a key factor to determine preferable slip and crack transfer. This local information is potentially important in predicting microstructural grain boundary behaviour and hence influencing crack growth. 
Therefore, the microstructural crack propagation which takes account of local crystal plasticity and dislocation accumulation maybe of importance and need to be addressed using full slip anisotropy with crystal plasticity. This approach has been used in other studies [28, 29] but using a non-local damage modelling technique to attempt to address local defect nucleation.

\section{Conclusion}

Several modelling techniques have been used and assessed to capture the experimental microstructurally-sensitive cracking witnessed in notched polycrystal specimens. Based on anisotropic elasticity with X-FEM crack propagation only, it was found for the six microstructurally different ferritic notched specimens considered that the X-FEM modelling captured the experimental observations for three specimens including the transgranular crack kinking and deviations observed.

A further two experimental specimens showed similar microstructural crack growth characteristics with both intergranular and transgranular behaviour as witnessed from SEM images. However intergranular crack growth features were found to be difficult to capture with anisotropic elasticity and X-FEM. To address this, the elastically anisotropic X-FEM models were enhanced with cohesive interfaces to allow crack growth and grain boundary separation to occur, though this allowed intergranular cracking to be captured only for the primary grain. A further methodology examined introduced explicit boundary zones along grain boundaries allowing grain boundary properties to be specified. Both elastically stiff and compliant grain boundaries were considered, and it was found that strong grain boundary properties allowed the quantitative prediction of the transition from transgranular to intergranular crack growth and the experimentally observed tortuous crack paths to be captured. Proposed studies for the future include consideration of local slip and dislocation activity which may lead to improved predictive capability for microstructurally-sensitive crack path prediction when coupled with X-FEM crack propagation modelling.

\section{Acknowledgement}

This work is part of a Collaborative R\&T Project, SILOET II, Project 10, Virtual Engine Design System, supported by the Technology Strategy Board and carried out by Rolls-Royce plc and Imperial College London. 


\section{Appendix A. SUS430LX ferritic steel microstructure characterisation}

Appendix table contains EBSD extracted data of each crystallographic grain orientation within the notch region of interested for six ferritic steel specimens, listed on Table 2. The three average orientations of a grain crystal follow Bunge's convention. NSC carried out the notch microstructure characterisation.

\begin{tabular}{|c|c|c|c|c|c|c|c|c|c|}
\hline \multirow{2}{*}{$\begin{array}{c}\text { Ferritic steel } \\
\text { sample ID }\end{array}$} & \multirow{2}{*}{ Grain ID } & \multicolumn{3}{|c|}{ Average orientation (Deg) } & \multirow{2}{*}{$\begin{array}{c}\text { Ferritic steel } \\
\text { sample ID }\end{array}$} & \multirow{2}{*}{ Grain ID } & \multicolumn{3}{|c|}{ Average orientation (Deg) } \\
\hline & & $\varphi_{1}$ & $\Phi$ & $\varphi_{2}$ & & & $\varphi_{1}$ & $\Phi$ & $\varphi_{2}$ \\
\hline \multirow{9}{*}{ SUS430LX_06 } & A & 298.29 & 27.94 & 87.48 & \multirow{11}{*}{ SUS430LX_11 } & A & 223.95 & 26.49 & 154.73 \\
\hline & $\mathrm{B}$ & 36.33 & 11.28 & 320.19 & & $\mathrm{~B}$ & 313.47 & 21.66 & 3.91 \\
\hline & c & 160.10 & 23.34 & 211.11 & & c & 104.21 & 37.11 & 247.91 \\
\hline & D & 94.39 & 11.03 & 291.10 & & D & 238.64 & 19.14 & 147.23 \\
\hline & $\mathrm{E}$ & 123.59 & 22.55 & 248.06 & & $\mathrm{E}$ & 243.00 & 43.04 & 114.77 \\
\hline & $\mathrm{F}$ & 330.79 & 21.65 & 83.92 & & $\mathrm{~F}$ & 141.90 & 15.11 & 218.47 \\
\hline & G & 52.97 & 31.36 & 331.25 & & G & 226.15 & 17.63 & 116.59 \\
\hline & $\mathrm{H}$ & 115.88 & 14.80 & 270.81 & & $\mathrm{H}$ & 70.24 & 28.76 & 290.86 \\
\hline & 1 & 27.72 & 40.01 & 289.70 & & $\mathrm{~K}$ & 233.56 & 48.88 & 138.18 \\
\hline \multirow{15}{*}{ SUS430LX_08 } & 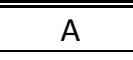 & 174.41 & 499.00 & 185.05 & & 1 & 24.80 & 3.61 & 351.53 \\
\hline & $\mathrm{B}$ & 214.83 & 29.78 & 146.15 & & $\mathrm{~J}$ & 181.27 & 24.55 & 143.62 \\
\hline & $\mathrm{C}$ & 66.19 & 44.31 & 294.45 & \multirow{12}{*}{ SUS430LX_12 } & A & 270.62 & 29.46 & 121.25 \\
\hline & D & 182.46 & 8.02 & 176.07 & & B & 40.62 & 33.44 & 273.30 \\
\hline & $\mathrm{E}$ & 45.72 & 44.93 & 323.24 & & C & 144.66 & 17.06 & 230.12 \\
\hline & $\mathrm{F}$ & 58.63 & 49.28 & 310.65 & & $\mathrm{D}$ & 285.49 & 41.88 & 56.45 \\
\hline & G & 189.30 & 23.46 & 195.93 & & $\mathrm{E}$ & 329.50 & 41.74 & 29.02 \\
\hline & $\mathrm{H}$ & 132.76 & 11.71 & 217.93 & & $\mathrm{~F}$ & 295.28 & 28.30 & 57.52 \\
\hline & $\mathrm{I}$ & 348.25 & 12.52 & 36.60 & & G & 129.65 & 29.97 & 230.68 \\
\hline & $\mathrm{J}$ & 158.26 & 47.70 & 238.60 & & $\mathrm{H}$ & 268.53 & 15.29 & 95.22 \\
\hline & $\mathrm{K}$ & 186.45 & 15.11 & 143.62 & & I & 129.39 & 29.84 & 230.42 \\
\hline & $\mathrm{L}$ & 189.36 & 9.64 & 207.40 & & $\mathrm{~J}$ & 156.54 & 38.23 & 189.90 \\
\hline & $\mathrm{M}$ & 52.21 & 46.65 & 331.49 & & $\mathrm{~K}$ & 292.43 & 37.53 & 45.16 \\
\hline & $\mathrm{N}$ & 149.99 & 29.44 & 194.18 & & $\mathrm{M}$ & 271.82 & 39.89 & 92.28 \\
\hline & 0 & 125.24 & 26.16 & 232.26 & \multirow{14}{*}{ SUS430LX_15 } & A & 201.37 & 35.91 & 157.46 \\
\hline \multirow{11}{*}{ SUS430LX_10 } & $\mathrm{A}$ & 236.65 & 23.61 & 123.72 & & $\mathrm{~B}$ & 110.51 & 18.73 & 262.51 \\
\hline & $\mathrm{B}$ & 197.28 & 38.32 & 189.67 & & C & 101.39 & 14.65 & 280.53 \\
\hline & C & 111.53 & 8.16 & 307.75 & & D & 194.31 & 67.49 & 196.29 \\
\hline & $\mathrm{D}$ & 302.46 & 24.70 & 19.60 & & $\mathrm{~F}$ & 85.59 & 12.07 & 280.35 \\
\hline & $\mathrm{E}$ & 202.65 & 20.99 & 155.20 & & $\mathrm{E}$ & 46.24 & 46.67 & 306.80 \\
\hline & $\mathrm{F}$ & 294.48 & 17.85 & 33.48 & & G & 106.81 & 9.65 & 280.42 \\
\hline & G & 295.10 & 17.60 & 32.73 & & $\mathrm{H}$ & 166.09 & 20.84 & 215.99 \\
\hline & $\mathrm{H}$ & 222.96 & 34.66 & 142.05 & & 1 & 9.49 & 19.44 & 12.10 \\
\hline & I & 186.10 & 27.81 & 178.38 & & J & 146.82 & 19.83 & 248.27 \\
\hline & $\mathrm{J}$ & 28.34 & 18.46 & 8.29 & & $\mathrm{k}$ & 2.09 & 20.47 & 19.96 \\
\hline & $\mathrm{K}$ & 116.31 & 7.16 & 212.74 & & $\mathrm{~L}$ & 322.40 & 17.50 & 30.02 \\
\hline & & & & & & $\mathrm{M}$ & 308.53 & 15.31 & 63.89 \\
\hline & & & & & & $\mathrm{N}$ & 163.73 & 32.25 & 245.35 \\
\hline
\end{tabular}




\section{References}

[1] A.A. Griffith, The phenomena of rupture and flow in solids, Philosophical transactions of the royal society of london. Series A, containing papers of a mathematical or physical character, 221 (1921) 163-198.

[2] G.R. Irwin, Analysis of stresses and strains near the end of a crack traversing a plate, J. Appl. Mech., (1957).

[3] T.N. Bittencourt, P.A. Wawrzynek, A.R. Ingraffea, J.L. Sousa, Quasi-automatic simulation of crack propagation for 2D LEFM problems, Engineering Fracture Mechanics, 55 (1996) 321-334.

[4] B.J. Carter, P.A. Wawrzynek, A.R. Ingraffea, Automated 3-D crack growth simulation, International Journal for Numerical Methods in Engineering, 47 (2000) 229-253.

[5] S. Mellings, J. Baynham, R.A. Adey, Predicting residual strength using fully automatic crack growth, Engineering Analysis with Boundary Elements, 26 (2002) 479-488.

[6] M. Schöllmann, M. Fulland, H.A. Richard, Development of a new software for adaptive crack growth simulations in 3D structures, Engineering Fracture Mechanics, 70 (2003) 249-268.

[7] M. Schöllmann, H.A. Richard, G. Kullmer, M. Fulland, A New Criterion for the Prediction of Crack Development in Multiaxially Loaded structures, International Journal of Fracture, 117 (2002) 129141.

[8] C.Timbrell, G. Cook, 3D FE fracture mechanics analysis for industrial applications, in: Seminar on Inelastic Finite Element Anlaysis, Institute of Mechanical Engineers, (1997).

[9] E. Giner, N. Sukumar, J.E. Tarancon, F.J. Fuenmayor, An Abaqus implementation of the extended finite element method, Engineering fracture mechanics, 76 (2009) 347-368.

[10] N. Sukumar, J. Dolbow, A. Devan, J. Yvonnet, F. Chinesta, D. Ryckelynck, P. Lorong, I. Alfaro, M. Martínez, E. Cueto, Meshless methods and partition of unity finite elements, International Journal of Forming Processes, 8 (2005) 409.

[11] N. Sukumar, D.J. Srolovitz, Finite Element-Based Model for Crack Propagation in Polycrystalline Materials, Computational \& Applied Mathematics, 23 (2004) 363-380.

[12] N. Sukumar, Z.Y. Huang, J.H. Prévost, Z. Suo, Partition of unity enrichment for bimaterial interface cracks, International Journal for Numerical Methods in Engineering, 59 (2004) 1075-1102.

[13] N. Moës, N. Sukumar, B. Moran, T. Belytschko, An extended finite element method (X-FEM) for two- and three-dimensional crack modeling, in: European Congress on Computational Methods in Applied Sciences and Engineering, Barcelona, Spain, 2000.

[14] B.Y. Chen, S.T. Pinho, P.M. Baiz, N.W.D. Carvalho, T.E. Tay, A floating node method for the modelling of discontinuities within a finite element, in: International Journal for Numerical Methods in Engineering, 2013.

[15] E. Barbieri, N. Petrinic, M. Meo, V. Tagarielli, A new weight - function enrichment in meshless methods for multiple cracks in linear elasticity, International Journal for Numerical Methods in Engineering, 90 (2012) 177-195.

[16] L. Saucedo-Mora, T.J. Marrow, FEMME: a multi-scale Finite Element Microstructure Meshfree fracture model for quasi-brittle materials with complex microstructures, Engineering Fracture Mechanics, 147 (2015) 355-372.

[17] T. Nguyen, J. Yvonnet, Q.-Z. Zhu, M. Bornert, C. Chateau, A phase field method to simulate crack nucleation and propagation in strongly heterogeneous materials from direct imaging of their microstructure, Engineering Fracture Mechanics, 139 (2015) 18-39.

[18] K. Hussain, Short fatigue crack behaviour and analytical models a review, Engineering Fracture Mechanics, Vol. 58 (1997) 327-354.

[19] K. Tokaji, T. Ogawa, The Growth Behaviour of Microstructurally Small Fatigue Cracks in Metals, Mechanical Engineering Publications, (1992) 85-99.

[20] F.P.E. Dunne, Fatigue crack nucleation: Mechanistic modelling across the length scales, Current Opinion in Solid State and Materials Science, Vol. 18, (2014), 170-179. 
[21] H.J. Christ, C.P. Fritzen, P. Köster, Micromechanical modeling of short fatigue cracks, Current Opinion in Solid State and Materials Science, 18 (2014) 205-211.

[22] H.J. Christ, O. Düber, C.P. Fritzen, H. Knobbe, P. Köster, U. Krupp, B. Künkler, Propagation behaviour of microstructural short fatigue cracks in the high-cycle fatigue regime, Computational Materials Science, 46 (2009) 561-565.

[23] P. Alveen, D. McNamara, D. Carolan, N. Murphy, A. Ivanković, The influence of microstructure on the fracture properties of polycrystalline cubic boron nitride, Computational Materials Science, 109 (2015) 115-123.

[24] A.C. Magee, L. Ladani, Representation of a microstructure with bimodal grain size distribution through crystal plasticity and cohesive interface modeling, Mechanics of Materials, 82 (2015) 1-12.

[25] P. Zhang, M. Karimpour, D. Balint, J. Lin, D. Farrugia, A controlled Poisson Voronoi tessellation for grain and cohesive boundary generation applied to crystal plasticity analysis, Computational Materials Science, 64 (2012) 84-89.

[26] H. Li, H. Yuan, Cohesive zone modelling of low cycle fatigue cracks in cracked and notched specimens, Fatigue \& Fracture of Engineering Materials \& Structures, 36 (2013) 1246-1257.

[27] D. Kupka, N. Huber, E. Lilleodden, A combined experimental-numerical approach for elastoplastic fracture of individual grain boundaries, Journal of the Mechanics and Physics of Solids, 64 (2014) 455-467.

[28] C. Madrigal, F.P.E. Dunne, M.R. Bache, Experimental and crystal plasticity studies of deformation and crack nucleation in a titanium alloy, The Journal of Strain Analysis for Engineering Design, 45 (2010) 391-399.

[29] F.P.E. Dunne, D. Rugg, On the mechanisms of fatigue facet nucleation in titanium alloys, Fatigue \& Fracture of Engineering Materials \& Structures, 31 (2008) 949-958.

[30] M. Sistaninia, M. Niffenegger, Prediction of damage-growth based fatigue life of polycrystalline materials using a microstructural modeling approach, International Journal of Fatigue, 66 (2014) 118-126.

[31] N. Vajragupta, V. Uthaisangsuk, B. Schmaling, S. Münstermann, A. Hartmaier, W. Bleck, A micromechanical damage simulation of dual phase steels using XFEM, Computational Materials Science, 54 (2012) 271-279.

[32] N. Sukumar, D.J. Srolovitz, T.J. Baker, J.H. Prevost, Brittle fracture in polycrystalline microstructures with the extended finite element method, International Journal for Numerical Methods in Engineering, 56 (2003) 2015-2037.

[33] K. Farhangdoost, S. Rahnama, Special criterion for crack path prediction at micro-structural scale based on dominate slip system and grain boundary, International Journal of Fatigue, 69 (2014) 49-62.

[34] A. Menk, S.P.A. Bordas, Crack growth calculations in solder joints based on microstructural phenomena with X-FEM, Computational Materials Science, 50 (2011) 1145-1156.

[35] V.V.C. Wan, D.W. MacLachlan, F.P.E. Dunne, A stored energy criterion for fatigue crack nucleation in polycrystals, International Journal of Fatigue, 68 (2014) 90-102.

[36] C.A. Sweeney, W. Vorster, S.B. Leen, E. Sakurada, P.E. McHugh, F.P.E. Dunne, The role of elastic anisotropy, length scale and crystallographic slip in fatigue crack nucleation, Journal of the Mechanics and Physics of Solids, 61 (2013) 1224-1240.

[37] Abaqus, Analysis User's Manual v6.11.

[38] J. Shi, D. Chopp, J. Lua, N. Sukumar, T. Belytschko, Abaqus Implementation of Extended Finite Element Method Using a Level Set Representation for Three-Dimensional Fatigue Crack Growth and Life Predictions, Engineering fracture mechanics, 77 (2010) 2840-2863.

[39] S. E. Mousavi, E. Grinspun, N. Sukumar, Higher-order extended finite elements with harmonic enrichment functions for complex crack problems, International Journal for Numerical Methods in Engineering, 86 (2011) 560-574. 
[40] D.Q. Shi, X.A. Hu, J.K. Wang, H.C. Yu, X.G. Yang, J. Huang, Effect of notch on fatigue behaviour of a directionally solidified superalloy at high temperature, Fatigue \& Fracture of Engineering Materials \& Structures, 36 (2013) 1288-1297.

[41] Y. Yang, N. Yao, B. Imasogie, W.O. Soboyejo, Nanoscale and submicron fatigue crack growth in nickel microbeams, Acta Materialia, 55 (2007) 4305-4315.

[42] S. Yuan, Y. Wang, D. Wei, Experimental investigation on low cycle fatigue and fracture behaviour of a notched $\mathrm{Ni}$ - based superalloy at elevated temperature, Fatigue \& Fracture of Engineering Materials \& Structures, 37 (2014) 1002-1012.

[43] K.-H. Schwalbe, Some aspects of crack propagation under monotonic and cyclic load, Engineering Fracture Mechanics, 9 (1977) 547-556.

[44] J. Newman Jr, Finite-element analysis of crack growth under monotonic and cyclic loading, ASTM STP, 637 (1977) 56-80.

[45] J.D. Carroll, W. Abuzaid, J. Lambros, H. Sehitoglu, High resolution digital image correlation measurements of strain accumulation in fatigue crack growth, International Journal of Fatigue, 57 (2013) 140-150.

[46] F. Farukh, L. Zhao, R. Jiang, P. Reed, D. Proprentner, B. Shollock, Realistic microstructure-based modelling of cyclic deformation and crack growth using crystal plasticity, Computational Materials Science, 111 (2016) 395-405.

[47] B. Lin, L. Zhao, J. Tong, A crystal plasticity study of cyclic constitutive behaviour, crack-tip deformation and crack-growth path for a polycrystalline nickel-based superalloy, Engineering Fracture Mechanics, 78 (2011) 2174-2192.

[48] M. Kartal, M. Cuddihy, F. Dunne, Effects of crystallographic orientation and grain morphology on crack tip stress state and plasticity, International Journal of Fatigue, 61 (2014) 46-58.

[49] D. Gonzalez, I. Simonovski, P. Withers, J.Q. da Fonseca, Modelling the effect of elastic and plastic anisotropies on stresses at grain boundaries, International Journal of Plasticity, 61 (2014) 4963.

[50] J. Jiang, T.B. Britton, A.J. Wilkinson, Mapping type III intragranular residual stress distributions in deformed copper polycrystals, Acta Materialia, 61 (2013) 5895-5904.

[51] A.F. Knorr, M. Marx, Calculating the Resistance of a Grain Boundary against Fatigue Crack Growth, Advanced Materials Research, 891 (2014) 929-935. 


\section{Figures}

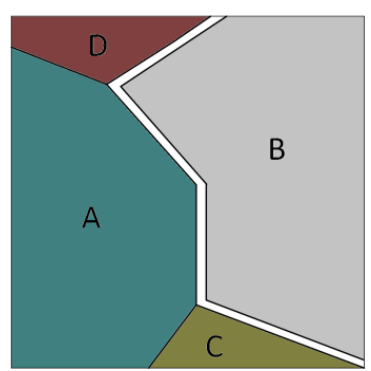

Fig. 1 - Schematic representation of selected grain boundary around grain B with discrete boundary element zones with a finite thickness

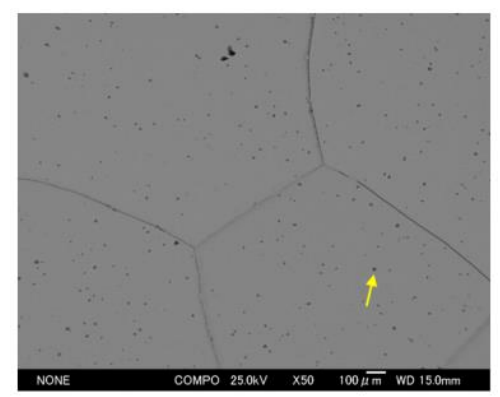

Fig. 2 - Back-Scattered Electron Image of single phase $\alpha$-Fe with TiN precipiates indicated by the yellow arrow

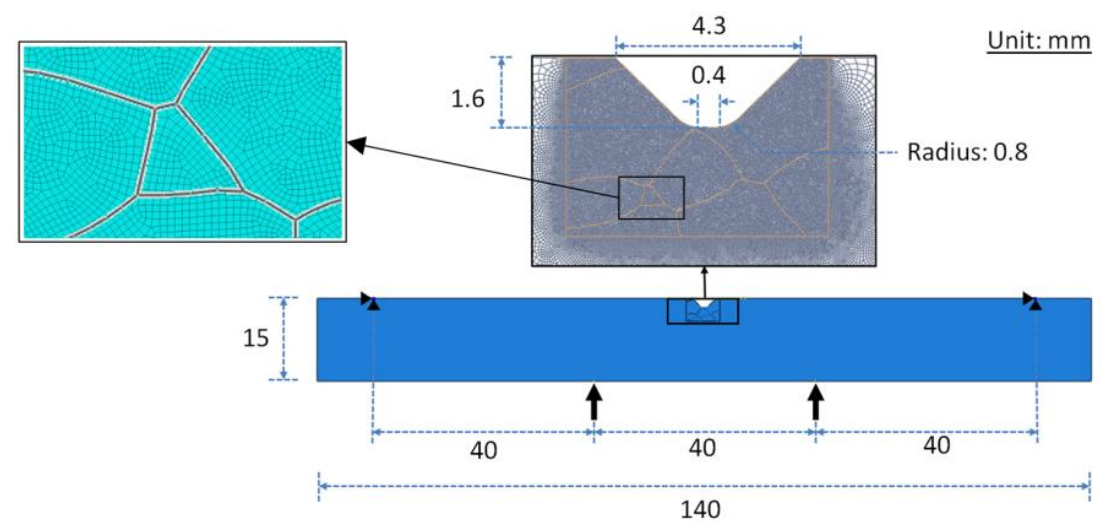

Fig. 3 - Geometry of the notched ferritic steel polycrystal under four-point bend [36] with explicit microstructure representation of densely meshed region around the notch 
(a)

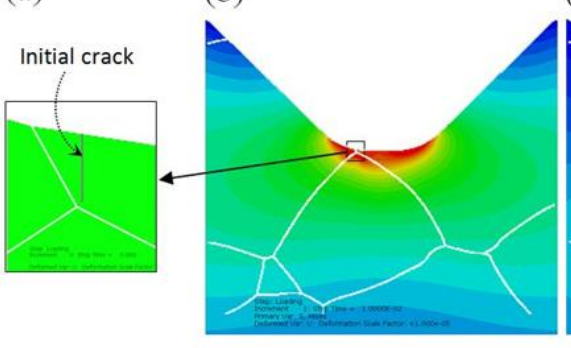

(c)

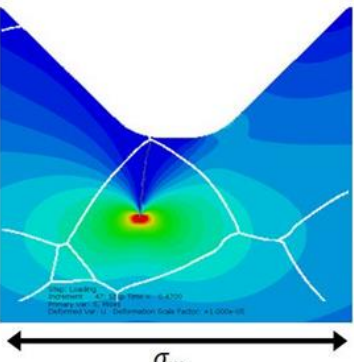

(d)

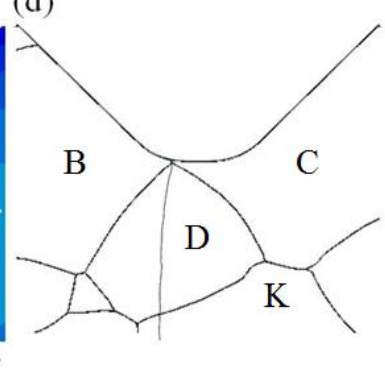

(e)

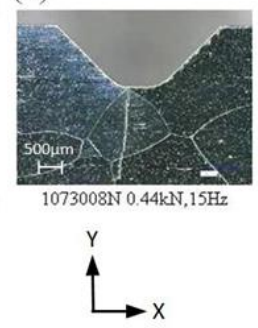

Fig. 4 - Isotropic elasticity with X-FEM sample A microstructure crack modelling (a) initial crack introduced in the model, (b) first incremental loading axial X-stress distribution, (c) axial stress distribution mid-crack growth in primary grain D, (d) final calculated crack path, and (e) SEM image of experimental crack propagation path. Note: in the model stress distribution contours, red indicates high stress, green medium stress and blue-low stress magnitudes.

(a)

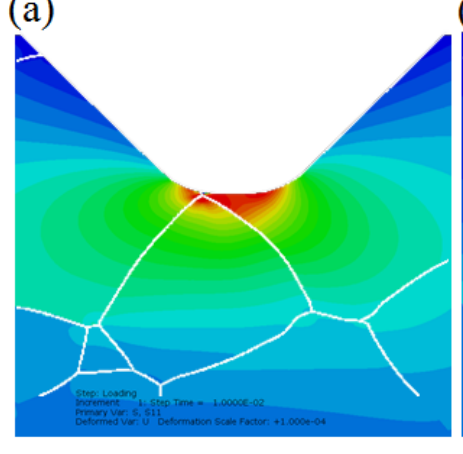

(b)

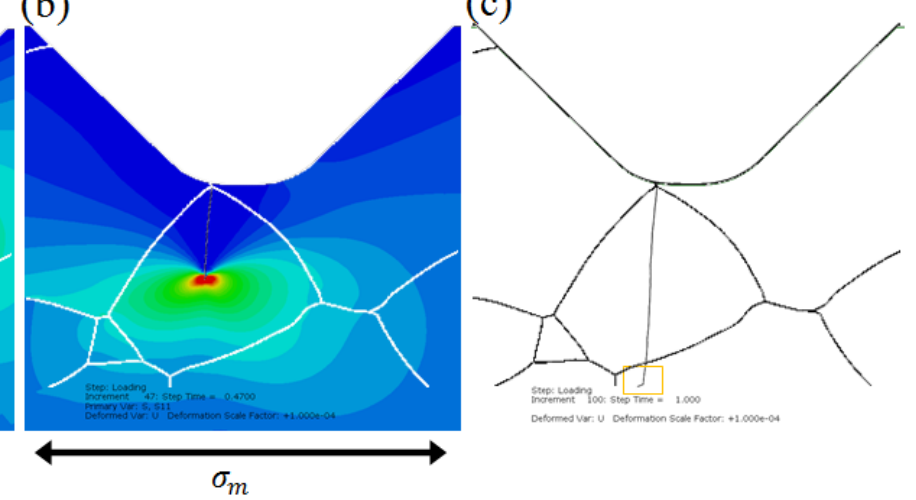

(d)
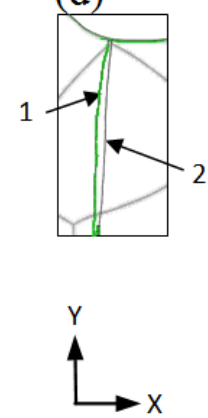

Fig. 5 - Anisotropic elasticity with X-FEM sample A microstructure crack modelling (a) first loading showing axial stress distribution, (b) axial stress distribution mid-crack growth in primary grain $D$, (c) final predicted crack path, and (d) comparing elastically isotropic crack solution ' 1 ' to anisotropic elasticity crack solution ' 2 '

(a)

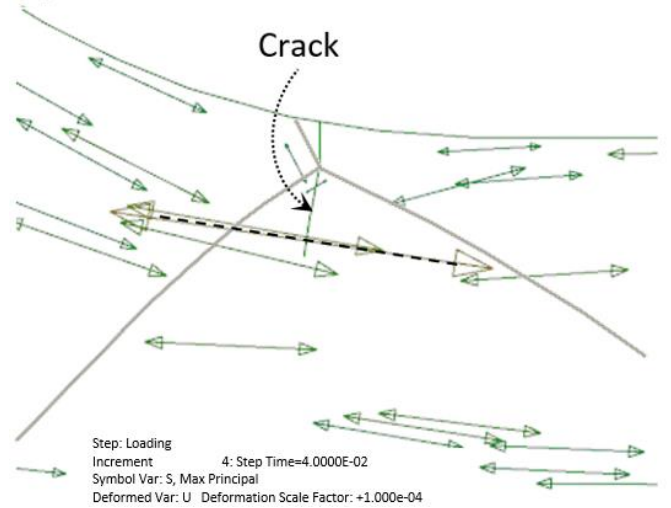

(b)

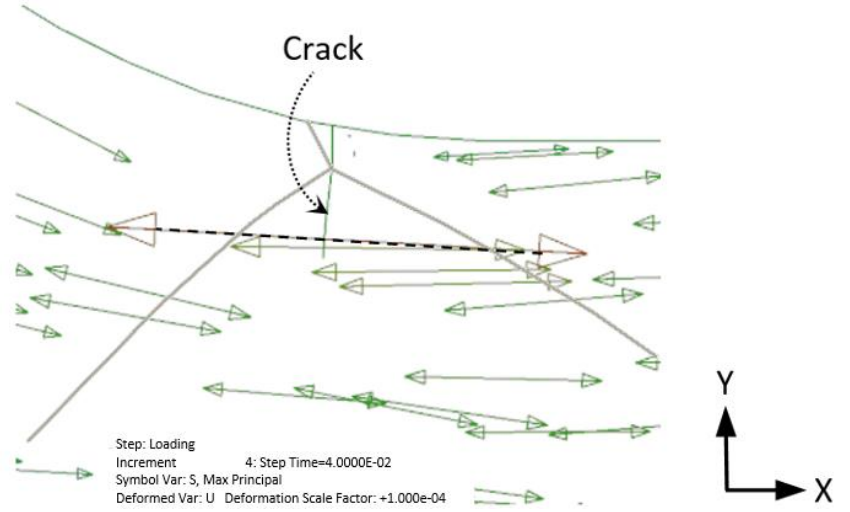

Fig. 6 - Distribution of maximum principal stress directions locally around the crack tip for (a) isotropic elasticity and (b) elastically anisotropic grains 

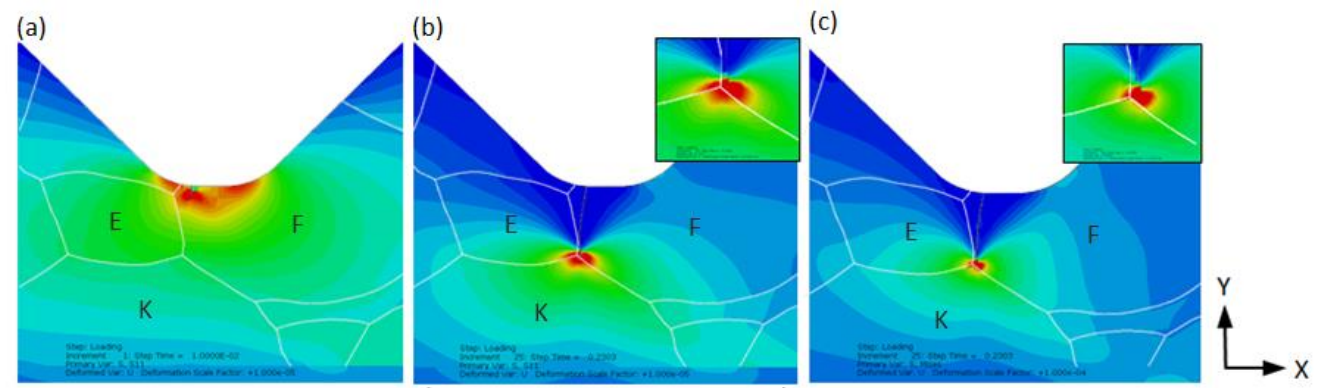

Fig. 7 - Elastically anisotropic X-FEM crack propagation for sample F showing (a) axial stress for the initial crack and loading state, and (b) subsequent crack growth up to grain boundary K-F and (c) Von Mises stress distribution for the same crack growth

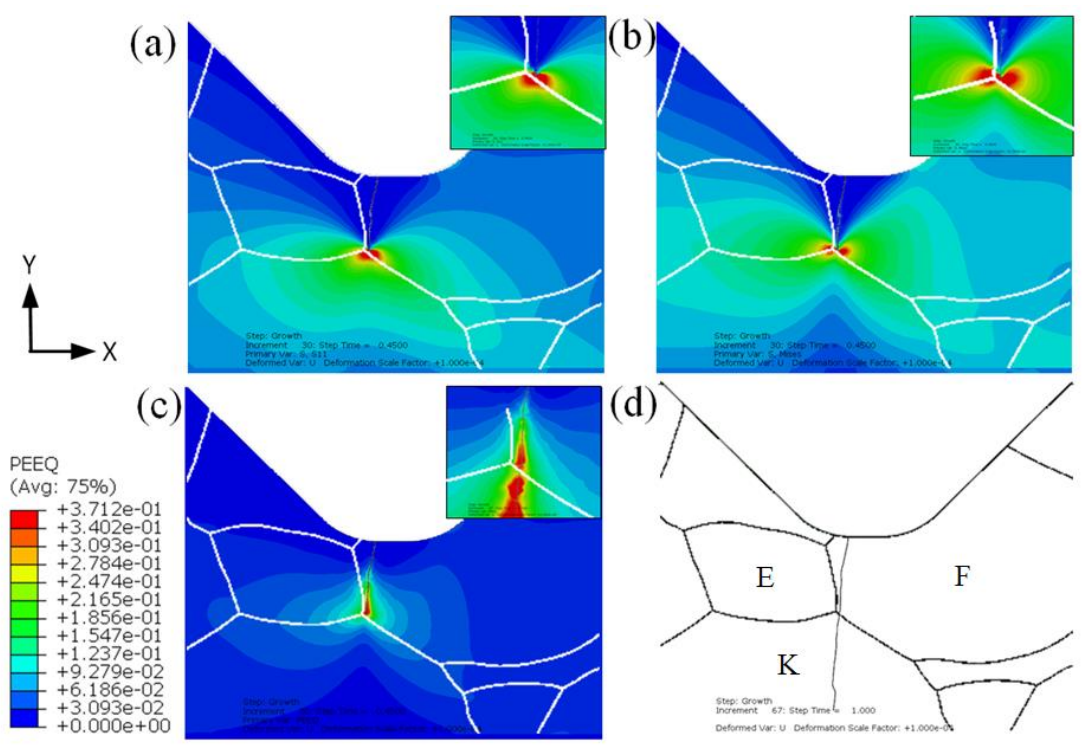

Fig. 8 - Anisotropic elasticity and isotropic plasticity X-FEM crack propagation for sample F illustrating crack growth up to grain boundary K-F with (a) axial stress distribution, (b) von Mises stress distribution, (c) equivalent plastic strain distribution and (d) final predicted microstructural crack growth path

(a)

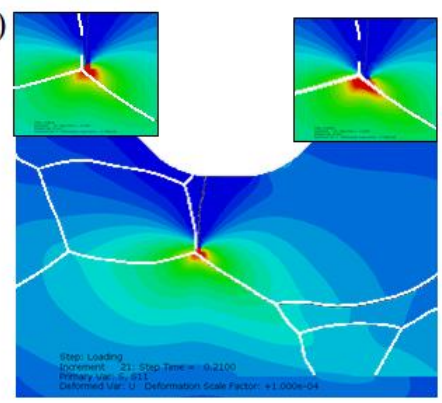

(b)

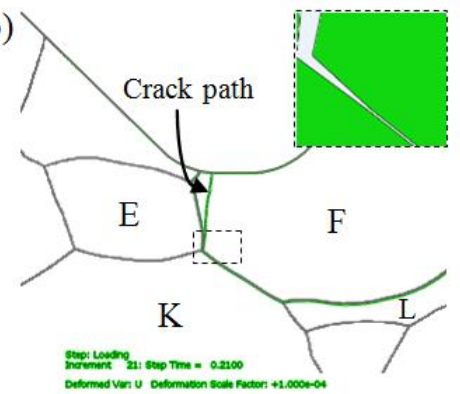

(c)

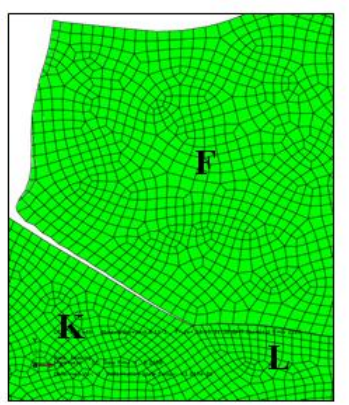

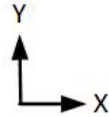

Fig. 9 - Cohesive-separation interfaces around grain F with X-FEM crack propagation (a) axial stress distribution with top left window showing crack at grain boundary K-F and top right initial separation 
along grain boundary and (b) commencement of grain boundary separation and intergranular crack growth, and (c) X-FEM/cohesive zone finite element model showing crack growth and grain boundary separation

(a)
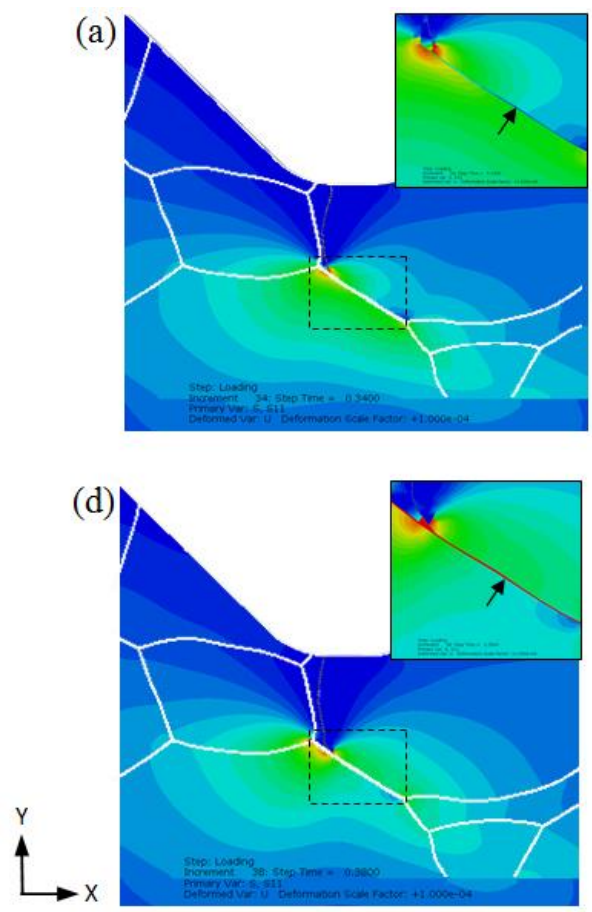

(b)

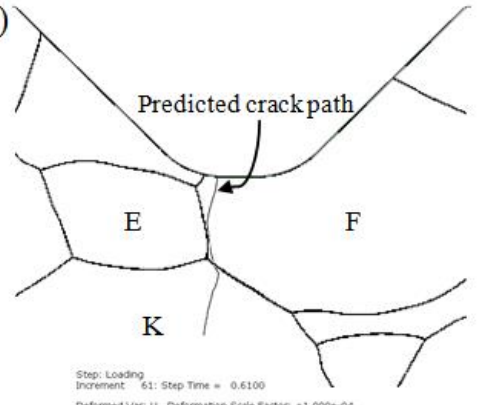

(e)

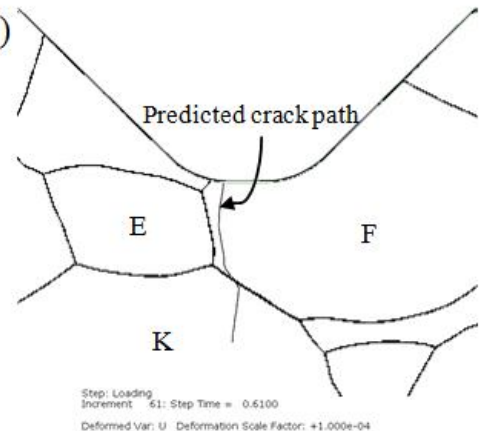

(c)

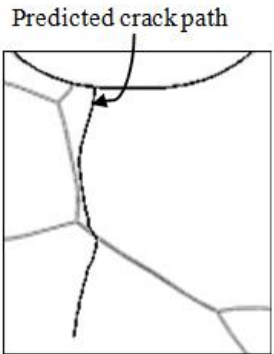

(f)

\section{Predicted crack path}

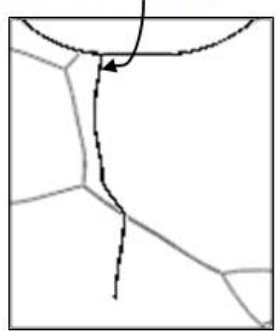

Fig. 10 - X-FEM crack propagation with grain boundary zone elements in sample F showing (a) axial stress distribution, (b) final predicted crack path and (c) enlarged predicted crack path with weak grain boundary properties, (d) axial stress distribution, (e) final predicted crack path and (f) enlarged predicted crack path with strong grain boundary properties 
(a)
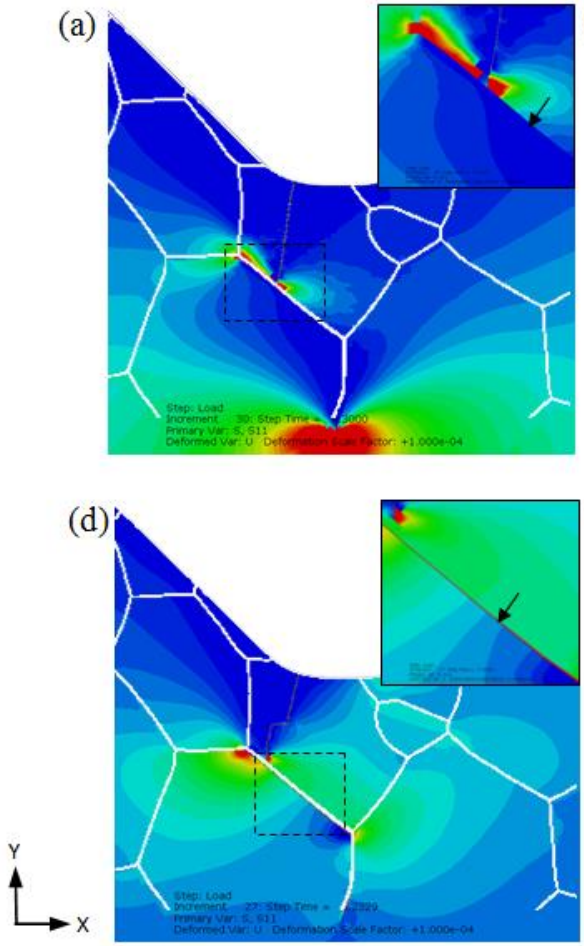

(b) $\lambda$

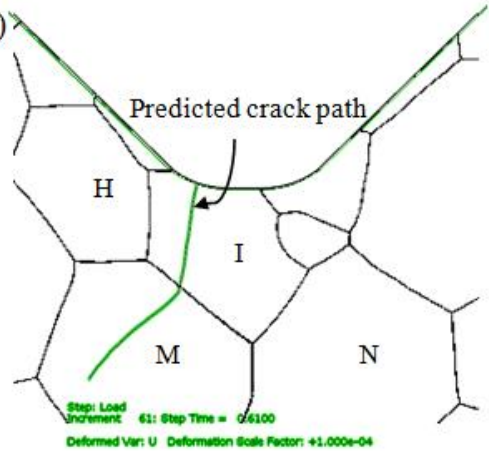

(e)

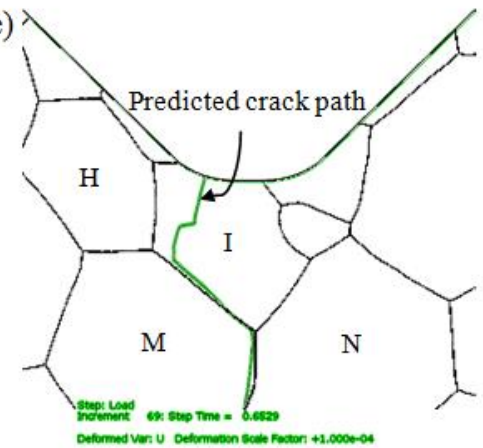

(c)
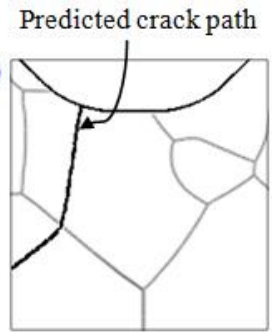

Predicted crack path

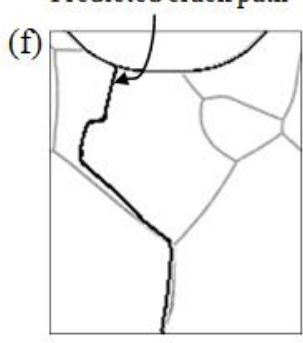

Fig. 11 - X-FEM crack propagation with boundary elements in sample B showing (a) axial stress distribution and (b) final predicted crack path and (c) enlarged predicted crack path with weak grain boundary properties, (d) axial stress distribution, (e) final predicted crack path and (f) enlarged predicted crack path with strong grain boundary properties. Note: the development of high stress at grain boundary M-N at the base of Fig. 11(a) is caused by the base boundary effect. 


\section{Tables}

Table 1 - SUS430LX Ferritic polycrystal steel composition

\begin{tabular}{|c|c|c|c|c|c|c|c|c|c|}
\hline \multirow{2}{*}{ Steel } & \multicolumn{10}{|c|}{ Chemical compositions (mass\%) } \\
\cline { 2 - 10 } & $\mathrm{C}$ & $\mathrm{Cr}$ & $\mathrm{Ni}$ & $\mathrm{P}$ & $\mathrm{S}$ & $\mathrm{Al}$ & $\mathrm{Ti}$ & $\mathrm{N}$ & $\mathrm{O}$ \\
\hline SUS430LX & 0.0007 & 16.37 & 0.1 & 0.031 & 0.0062 & 0.012 & 0.11 & 0.0363 & 0.0030 \\
\hline
\end{tabular}

Table 2 - Six different microstructure grain morphology within the notch region where detailed experimental fatigue crack data are presented in $[35,36]$

\begin{tabular}{|c|c|c|c|c|c|}
\hline $\begin{array}{c}\text { Ferritic Steel } \\
\text { ID }\end{array}$ & $\begin{array}{l}\text { Sample } \\
\text { ID }\end{array}$ & Morphology $2 \mathrm{~mm}$ & $\begin{array}{c}\text { Ferritic Steel } \\
\text { ID }\end{array}$ & $\begin{array}{c}\text { Sample } \\
\text { ID }\end{array}$ & $\begin{array}{l}\text { Morphology } \\
2 \mathrm{~mm}\end{array}$ \\
\hline SUS430LX_11 & $A$ & & SUS430LX_06 & D & \\
\hline SUS430LX_08 & B & & SUS430LX_12 & $E$ & \\
\hline SUS430LX_10 & C & A & SUS430LX_15 & $\mathrm{F}$ & \\
\hline
\end{tabular}


Table 3 - Full summary and assessment of the six differing microstructure morphology polycrystal samples based on anisotropic elasticity with X-FEM microstructure sensitive crack modelling

\begin{tabular}{|c|c|c|c|c|}
\hline Sample ID & SEM image & $\begin{array}{c}\text { Load / } \\
\text { Frequency }\end{array}$ & $\begin{array}{l}\text { Cycles to } \\
\text { failure }\end{array}$ & $\begin{array}{c}\text { XFEM } \\
\text { with full anisotropy }\end{array}$ \\
\hline A & & $\begin{array}{l}440 \mathrm{~N} / \\
15 \mathrm{~Hz}\end{array}$ & $1,073,008$ & \\
\hline B & & $\begin{array}{c}420 \mathrm{~N} / \\
15 \mathrm{~Hz}\end{array}$ & 241,161 & \\
\hline C & & $\begin{array}{l}440 \mathrm{~N} / \\
15 \mathrm{~Hz}\end{array}$ & $1,372,059$ & \\
\hline D & & $\begin{array}{c}400 \mathrm{~N} / \\
15 \mathrm{~Hz}\end{array}$ & 591,215 & \\
\hline $\mathrm{E}$ & & $\begin{array}{l}440 \mathrm{~N} / \\
15 \mathrm{~Hz}\end{array}$ & $1,276,428$ & \\
\hline $\mathrm{F}$ & & $\begin{array}{l}460 \mathrm{~N} / \\
15 \mathrm{~Hz}\end{array}$ & 321,573 & \\
\hline
\end{tabular}

